\title{
Casos clínicos en Cardiología (n.- 13): diagnóstico diferencial de la taquicardia de QRS ancho: a propósito de un caso
}

\author{
E. Heras Sánchez ${ }^{a}$, F. J. Pérez-Lescure Picarzo ${ }^{b}$ \\ ${ }^{a}$ MIR-Pediatría. Área de Pediatría. Hospital Universitario Fundación Alcorcón. Alcorcón, Madrid. España \\ - bCardiología Infantil. Área de Pediatría. Hospital Universitario Fundación Alcorcón. \\ Alcorcón, Madrid. España.
}

23-septiembre-2014

Francisco Javier Pérez-Lescure Picarzo: jplescure@fhalcorcon.es
Palabras clave:
- Taquicardia
supraventricular
- Taquicardia
ventricular

- Palpitaciones

- Cardioversión
Continuamos con la serie de casos clínicos en Cardiología pediátrica, revisando los motivos de consulta y datos exploratorios en las consultas de Pediatría de Atención Primaria presentados de forma breve y práctica. En este número se presenta una urgencia cardiológica en el centro de salud. Se trata de un niño de siete años que acude por palpitaciones de inicio brusco. Se discute cómo la historia, la exploración y los hallazgos electrocardiográficos nos orientan hacia el origen de una taquicardia que a su vez condicionará el tratamiento del paciente.

Key words:

- Supraventricular

tachycardia

- Ventricular

tachycardia

- Palpitations

- Cardioversion

\section{Clinical cases in Cardiology (No. 13): differential diagnosis of wide QRS tachycardia: a case report}

\begin{abstract}
We continue the series of clinical cases in Pediatric Cardiology by reviewing reasons for consultation in Primary Care and exploratory data, presented briefly and practically. In this issue a cardiological emergency occurs in the health center. It is a seven year old boy who presented with sudden onset palpitations. We discuss how the history, examination, and electrocardiographic findings point us to the origin of tachycardia, which in turn will influence the patient's treatment.
\end{abstract}

\section{CASO CLÍNICO}

Niño de siete años que acude a la consulta de Pediatría de Atención Primaria por presentar, 30 minutos antes y mientras caminaba, una sensación de palpitaciones de inicio brusco, sin dolor torácico o disnea acompañante. No había realizado ejercicio previamente. Sin antecedentes personales de cardiopatía, ni antecedentes familiares de enfermedades cardiacas o muerte súbita.

A su llegada al centro de salud, el paciente se encuentra consciente y orientado, sin signos de difi- cultad respiratoria. La auscultación cardiaca muestra tonos rítmicos a 170-180 latidos por minuto (lpm), sin soplos. Los pulsos periféricos son rítmicos y simétricos. Buen relleno capilar. Tensión arterial (TA) sistólica: $90 \mathrm{mmHg}$, percentil $31\left(\mathrm{P}_{31}\right)$, diastólica: $65 \mathrm{mmHg}\left(\mathrm{P}_{76}\right)$.

Se realiza un electrocardiograma (ECG) (Fig. 1). En la Fig. 2 se muestra la medición de la anchura del complejo QRS.

Ante los hallazgos electrocardiográficos de taquicardia de ORS ancho sin repercusión hemodinámica, su pediatra inicia perfusión de amiodarona

Cómo citar este artículo: Heras Sánchez E, Pérez-Lescure Picarzo FJ. Casos clínicos en Cardiología (n. $\left.{ }^{\circ} 13\right)$ : diagnóstico diferencial de la taquicardia de QRS ancho: a propósito de un caso. Rev Pediatr Aten Primaria. 2014;16:231-5. 


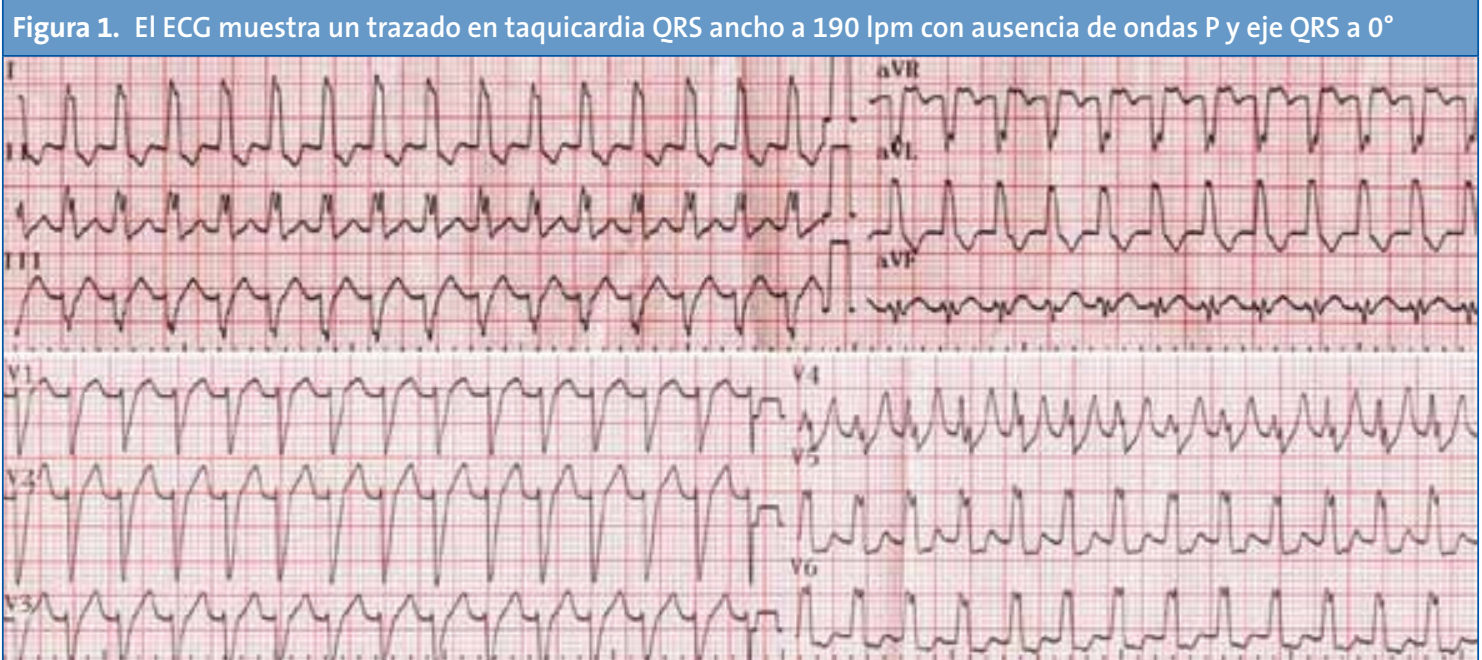

(5 mg/kg, vía intravenosa [IV]), y remite al paciente al hospital mediante transporte sanitario medicalizado. A su llegada al Servicio de Urgencias Pediátricas, el paciente estaba asintomático salvo por la sensación de palpitaciones. La exploración física fue la siguiente: peso $22,5 \mathrm{~kg}\left(\mathrm{P}_{21}\right)$, talla $119,5 \mathrm{~cm}\left(\mathrm{P}_{14}\right)$, TA sistólica $88 \mathrm{mmHg}\left(\mathrm{P}_{25}\right)$ y diastólica $63\left(\mathrm{P}_{71}\right)$. Consciente y orientado. Buena perfusión. Auscultación cardiaca con taquicardia sin soplos. Pulsos periféricos normales. Resto normal.

Tras el análisis del ECG, se considera la posibilidad de una taquicardia supraventricular (TSV) conducida con aberrancia, por lo que se decide intentar revertir la taquicardia con maniobras vagales (reflejo de náusea, frío en la cara), sin éxito, por lo que se administra un bolo de adenosina a 0,1 mg/kg IV

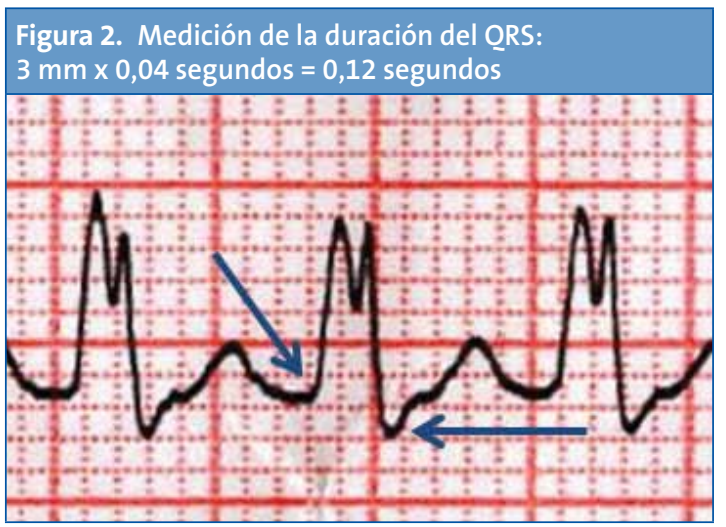

saliendo a ritmo sinusal (Fig. 3). Se realiza un ECG basal, que no muestra alteraciones (Fig. 4). La analítica sanguínea mostró iones y enzimas miocárdicas dentro del rango de la normalidad.

El paciente fue ingresado durante 24 horas para observación, sin presentar nuevos episodios, se realizó un ecocardiograma que resultó normal y fue dado de alta con posterior seguimiento en consultas de Cardiología, con diagnóstico de TSV paroxística con conducción aberrante.

\section{DISCUSIÓN}

La taquicardia en niños se define como una frecuencia cardiaca por encima de 180 lpm en lactantes y niños pequeños, y de 120 lpm en niños mayores y adolescentes.

Ante un niño con taquicardia, una vez descartada la presencia de repercusión hemodinámica que precise actuación inmediata, se debe analizar el ECG de 12 derivaciones para tratar de establecer el origen de la taquicardia, que nos permitirá guiar el tratamiento posterior. La anchura del complejo QRS nos permite una primera aproximación.

Las taquicardias con complejo QRS estrecho $(\leq 0,08$ segundos) son de origen supraventricular (originadas por encima o en el nódulo auriculoventricular $[\mathrm{AV}])$. Las taquicardias con complejos QRS anchos 
Figura 3. Reversión de la taquicardia

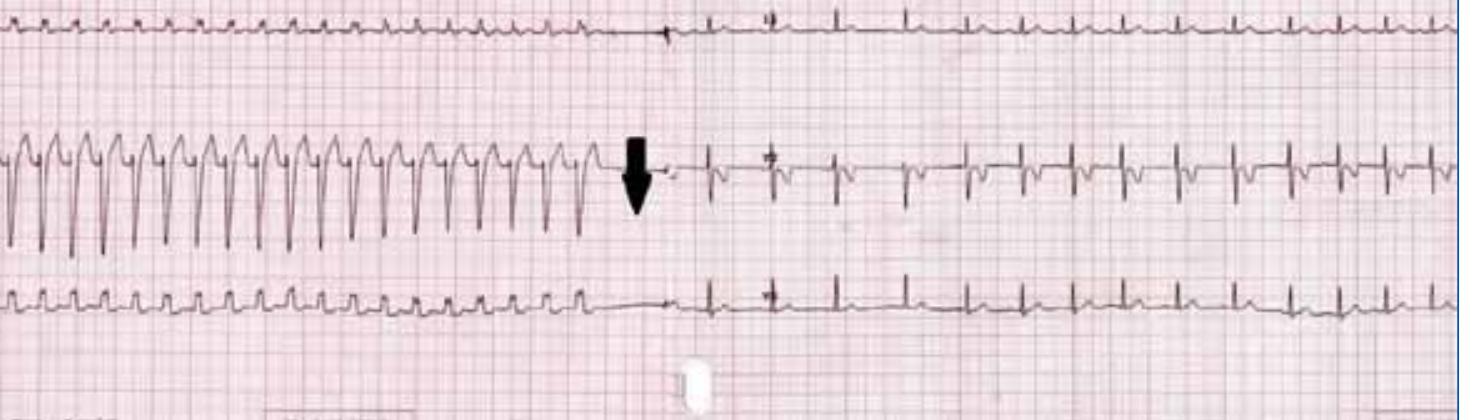

(>0,08 segundos) pueden originarse en los ventrículos (taquicardia ventricular [TV]), pero también pueden aparecer en pacientes con TSV con conducción aberrante (ya sea por la existencia de un bloqueo de rama o por la existencia de una vía accesoria auriculoventricular).

Ante una taquicardia de QRS ancho, la primera consideración debe ser siempre la TV, pues puede degenerar rápidamente en fibrilación ventriculary parada cardiaca.
Un diagnóstico correcto en cuanto al origen de la taquicardia es importante, debido a la necesidad de un tratamiento inmediato y diferente según sea el origen de la taquiarritmia, y al pronóstico más desfavorable de la TV, considerada una urgencia hospitalaria. De esta forma, un paciente con taquicardia de QRS ancho hemodinámicamente inestable debe ser tratado mediante cardioversión eléctrica de forma inmediata; sin embargo, cuando el paciente se encuentra estable, es preciso realizar el diagnóstico diferencial entre TSV conducida con aberrancia y TV.

Figura 4. ECG basal; ritmo sinusal a $105 \mathrm{lpm}$, onda P normal, intervalo PR 0,14 segundos, eje QRS a $30^{\circ}$, complejo QRS sin anomalías morfológicas, con duración de 0,06 segundos. Intervalo QTc de 0,42 segundos. Sin alteraciones de la repolarización

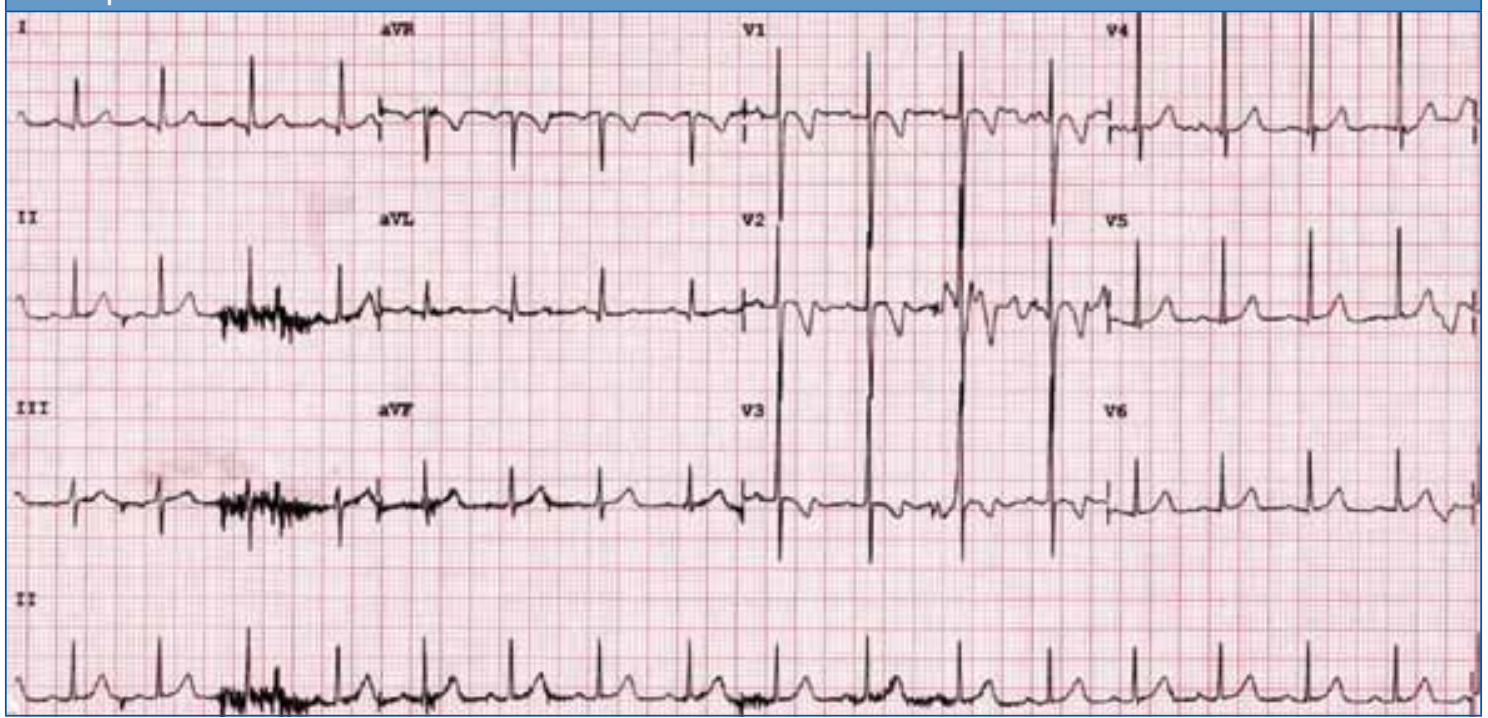


En las series más amplias publicadas de taquicardia de QRS ancho en niños ${ }^{1,2}$, la mayor parte de las taquicardias fueron de origen supraventricular, a diferencia de los adultos.

En la población infantil se deben utilizar una combinación de datos de la historia clínica, la exploración física y el análisis del ECG de 12 derivaciones para intentar identificar el origen de la taquicardia de QRS ancho.

La historia clínica debe dirigirse a identificar enfermedades cardiacas previas que puedan predisponer a la aparición de una TV, como cardiopatías congénitas, cirugía cardiaca reciente, canalopatías o prolongación adquirida del QT secundaria a fármacos tales como antiarrítmicos, macrólidos, antihistamínicos y ciertos antidepresivos o antipsicóticos.

Determinadas alteraciones metabólicas como la hipopotasemia o la hipomagnesemia pueden producir asimismo taquicardias ventriculares debidas al alargamiento del intervalo OT.

Finalmente, la presencia de antecedentes familiares de muerte súbita puede hacer sospechar condiciones hereditarias asociadas con TV, como el síndrome de OT largo congénito, el síndrome de Brugada o la displasia arritmogénica del ventrículo derecho.

La exploración física permite evaluar la estabilidad hemodinámica del paciente, que orientará la necesidad de tratamiento inmediato. Se debe considerar la cardioversión inmediata en los pacientes que presenten signos de dificultad respiratoria, disminución del nivel de consciencia, extremidades frías, relleno capilar prolongado o pulsos periféricos débiles.

La presencia de disociación AV es uno de los criterios más útiles para identificar la TV cuando está presente. EI ECG muestra la independencia de las ondas P y de los complejos QRS (ondas P a menor frecuencia del ritmo ventricular). Los latidos de captura (una onda P disociada activa el ventrículo) y los latidos de fusión (un estímulo sinusal y un estímulo ventricular coinciden para producir un complejo intermedio) pueden aparecer en la diso- ciación AV. La variabilidad de los complejos QRS puede resultar indicativa asimismo de TV. Sin embargo, no siempre se detecta disociación AV en las TV, al poder existir conducción retrógrada desde los ventrículos a las aurículas.

En las últimas décadas se han publicado distintos algoritmos para distinguir el origen de la taquicardia de ORS ancho en la población adulta ${ }^{3-7}$. Los algoritmos de Brugada y Vereckei han informado sensibilidades y especificidades en adultos de hasta el 96 y el 98\% para distinguir en el ECG de 12 derivaciones el origen supraventricular o ventricular de la taquicardia de QRS ancho ${ }^{4,7}$.

No obstante, estos algoritmos no han resultado de la misma utilidad en la población infantil ${ }^{1}$. Ceresnak et al., analizando 65 episodios de taquicardia de QRS ancho en 58 pacientes, encontraron que una desviación izquierda del eje y la presencia de una muesca en la rama descendente del complejo QRS eran más frecuentes en la TV que en la TSV. Por otro lado, la presencia de una deflexión positiva en V1 era más frecuente en la TSV.

La presencia de concordancia (complejos ORS monofásicos y de la misma polaridad en todas las derivaciones precordiales, positivos en V1-V6 con ondas R altas monofásicas, o negativos con complejos OS profundos) puede también orientar hacia el origen de la taquicardia ${ }^{8}$. La concordancia negativa es muy sugestiva de TV, la concordancia positiva aparece con más frecuencia en la TV, aunque puede también aparecer en algunas TSV antidrómicas. Disponer de un ECG previo en ritmo sinusal puede ser muy útil, pues si la configuración (morfología y eje) del QRS durante la taquicardia es idéntica al ritmo sinusal, ello sugiere un muy probable origen supraventricular.

Nuestro paciente se encontraba hemodinámicamente estable, lo cual permitió proceder al diagnóstico diferencial de la taquicardia de QRS ancho. En nuestro caso, no había datos en los antecedentes familiares y personales ni en la historia clínica que orientaran a un origen ventricular de la taquicardia. Respecto a los criterios electrocardiográficos, el trazado del ECG no mostraba disociación 
ventricular, el eje del ORS era normal y no existía concordancia. Los iones y enzimas cardiacas se encontraban dentro del rango de la normalidad.

\section{CONCLUSIÓN}

En muchas ocasiones, el diagnóstico diferencial entre TV y TSV conducida con aberrancia es difícil. En los niños, cuando la situación clínica lo permita, se deben utilizar datos de la historia, la exploración y el ECG para intentar establecer el origen de la taquicardia, que guiará el tratamiento.

Se debe estar familiarizado con los algoritmos utilizados en adultos, aunque no han demostrado la misma sensibilidad ni especificidad en niños.

\section{BIBLIOGRAFÍA}

1. Ceresnak SR, Liberman L, Avasarala K, Tanel R, Motonaga KS, Dubin AM. Are wide complex tachycardia algorithms applicable in children and patients with congenital heart disease? J Electrocardiol. 2010;43: 694-700.

2. Benson Jr. DW, Smith WM, Dunnigan A, Sterba R, Gallagher JJ. Mechanisms of regular, wide QRS tachycardia in infants and children. Am J Cardiol. 1982;49: 1778-88.

3. Akhtar M, Shenasa M, Jazayeri M, Caceres J, Tchou PJ. Wide ORS complex tachycardia. Reappraisal of a common clinical problem. Ann Intern Med. 1988; 109:905-12.

4. Brugada P, Brugada J, Mont L, Smeets J, Andries EW. A new approach to the differential diagnosis of a regu-
A efectos prácticos, ante una taquicardia con complejo QRS ancho en la edad pediátrica con el paciente hemodinámicamente estable y sin datos que orienten a un origen ventricular de la taquiarritmia, se puede iniciar tratamiento según el algoritmo de la TSV.

\section{CONFLICTO DE INTERESES}

Los autores declaran no presentar conflictos de intereses en relación con la preparación y publicación de este artículo.

\section{ABREVIATURAS}

AV: auriculoventricular • ECG: electrocardiograma • IV: vía intravenosa $\bullet$ Ipm: latidos por minuto $\bullet \mathrm{P}_{\mathrm{n}}$ : percentil $\mathrm{n} \bullet \mathrm{TA}$ : tensión arterial $\bullet$ TSV: taquicardia supraventricular $\bullet$ TV: taquicardia ventricular. lar tachycardia with a wide OQRS complex. Circulation. 1991;83:1649-59.

5. Griffith MJ, de Belder MA, Linker NJ, Ward DE, Camm AJ. Multivariate analysis to simplify the differential diagnosis of broad complex tachycardia. Br Heart J. 1991;66:166-74.

6. Kindwall KE, Brown J, Josephson ME. Electrocardiographic criteria for ventricular tachycardia in wide complex left bundle branch block morphology tachycardias. Am J Cardiol. 1988;61:1279-83.

7. Vereckei A, Duray G, Szenasi G, Altemose GT, Miller JM. Application of a new algorithm in the differential diagnosis of wide ORS complex tachycardia. Eur Heart J. 2007;28:589-600.

8. Gupta AK, Thakur RK. Wide ORS complex tachycardias. Med Clin North Am. 2001;85:245-66. 